\title{
THE CARE OF THE BLADDER IN TRAUMATIC PARAPLEGIA
}

\author{
By A. G. HARDY, M.B.E., B.M., B.Ch.
}

Consultant-in-charge, Spinal Injuries Unit, Sheffield

The treatment of traumatic paraplegia has undergone considerable changes since the period of the Second World War. The marked improvement in the results has been achieved by the better co-ordination of the management of all aspects of this still difficult problem, particularly in the special spinal centres in which the patients have been collected. It has been possible to establish a continuity of routine with appropriate emphasis on the handling of any phase in the disability at the right moment and concurrently with the general regime in progress at any one time.

Disordered bladder function is only one of the many problems presented by paraplegic patients, but the early treatment of the bladder is perhaps the most important aspect of the management of the patient as a whole. Nevertheless, it cannot be overemphasized that each problem of paraplegia, whether it be concerned with an orthopaedic lesion, the bladder, the bowels, the skin, the neuromuscular mechanisms or any complications attached thereto, is intimately bound up with each other and successful management of the whole depends on the adequate handling of each integral part.

There is no question whatsoever that bed sores, sepsis, muscle spasms, prolonged recumbency and immobility can all produce profound structural and functional changes in the urinary tract. Similarly, uncontrolled infection and complications in the urinary tract can predispose to complications appearing in other systems. It is because of this interrelationship of physiological and pathological mechanisms that one must be very careful not to over-concentrate on the treatment of one particular aspect to the extent of overlooking complications developing in another.

There are thus many factors which may have some bearing on the ultimate recovery of bladder function. Of those not directly concerned with the urinary tract itself the most important are related to the early general mobilization of the patient. The management of this part of the paraplegic programme reflects the greatest changes in the past decade. Whereas at one time the emphasis used to be on immobilization, it is now on early and general mobilization commencin within a matter of hours or days after the injury.

\section{The General Regime}

Orthopaedic Measures.-Specific orthopaed measures, if indicated, should be designed to assist in the easy handling of the patient and the prevention of further damage to the neural tissues. If stability is required for an unstable fracture dislocation it should be obtained by open reduc tion and internal fixation, particularly in the low dorso-lumbar injuries or skull traction in cervecal injuries. No paraplegic patient should be purin a plaster bed.

Nursing.-All cases should be turned and rolie in bed at two-hourly intervals day and nigh" This time interval can be increased according to the reaction in the skin and subcutaneous tissues This not only helps to protect the skin but als $\vec{\theta}$ to prevent stasis in the renal tract. The early nursing mobilization is continued with the eleva? tion of the patient in bed after four weeks, the attainment of the chair stage within a period of 8 to 12 weeks, and an ambulatory stage between. 12 and 16 weeks in most cases. This furthe assists drainage from the kidneys, but the nursing programme is paramount in the prevention of bed sores.

Physiotherapy.-Early active and passive physio therapy include spring expander exercises for the upper limbs and passive movements of the lowe These assist not only in the improvement of the fitness of the patient but also in the activity of the blood circulation throughout all areas. They als assist in the control of reflex mechanisms in fluencing somatic and autonomic systems alike.

Bowel Control.-This is all too often forgottet Inopportune incontinence and soiled linen, not mention chronic faecal retention with overflow, can be controlled by the judicious use of laxatives aperients, enemas or digital evacuation of the 
rectum, either singly or in combination, according to the return of reflex or other activity in the lower bowel.

Diet.-The normal diet should include a liberal fluid intake of between 5 and 6 pints daily. This is easily maintained by most patients.

\section{Neurological Considerations}

Following spinal cord injury the bladder mechanisms go into a phase of inactivity which may last from hours to months. This is followed by a period of increasing reflex activity when for long periods the reflex contractions are of insufficient strength or duration to produce efficient emptying of the bladder and the co-ordination of detrusor action and sphincter relaxation is very imperfect.'

This is the stage sometimes referred to as the autonomous bladder and is manifested clinically by an overflow, the volume of which can be increased by external influences such as manual compression of the abdomen or abdominal straining, or both. It may be the final bladder state in cases in which there has been complete destruction of the sacral segments of the cord or section of all the roots of the cauda-equina.

On the other hand, the reflex activity may increase until the reflex contractions are strong enough to produce a micturition characterized by forceful involuntary periodic voiding, the efficiency of which varies from case to case.

Co-ordinated reflex activity of this type depends for its existence on an intact sacral cord. Its appearance can be anticipated in some measure by the presence or absence of other reflexes using the same segmental pathways. These are the glans bulbar and anal skin reflexes and the ankle jerks. It can be observed in the first feeble efforts of urine voiding.

Interference in the co-ordination of reflex activity occurs when other reflex arcs influence the sacral segmental centres. The type of bladder function that can be obtained in any given case depends, firstly, on the integrity of the sacral segmental centres, and secondly, on the influence of other reflex centres particularly at a higher level in the cord but below the level of any given lesion.

It is therefore important to know something about the state of the cord below the level of the injury so that adverse reflexes may be anticipated and favourable ones encouraged. The impulses which occur during mass withdrawal responses spread over a wide area. The greater the number of segmental areas that are contained in the isolated cord the greater the diffusion of reflex activity. Even isolated sacral segments alone can produce sufficient adverse disturbance to upset bladder function merely by spasm of the perineal floor which also includes the external sphincter. Indeed, both clonic and tonic spasm of the external sphincter is a common occurrence in all reflex cord bladders, and uncontrolled somatic spasm in the locomotor systems is a very common factor in upsetting reflex bladder function. The control of spastic plantar flexion of the feet, adduction and flexion of the hips, flexion of the knees, either by remedial exercise, neurectomy or tenotomy or by all three measures together, is reflected not only in the muscles themselves but in the changes of reflex bladder mechanisms influenced by the central spread of nerve impulses. In some extremes the conversion of an uncontrolled spastic lesion to a flaccid one by means of an alcohol block can produce an efficient autonomous bladder action in response to abdominal pressure in place of a highly unbalanced reflex cord action.

\section{Management of the Bladder}

The aim of all management is to prevent distension and infection and restore function to a stage of efficient voiding with as much control as possible. This demands care and attention over potentially long periods with the object of keeping infection of both the upper and lower urinary tract to a minimum, avoiding genital sepsis and allowing frequent and easy observation of the state of activity of the bladder musculature. Treatment is designed primarily to prevent complications, and secondly, to encourage and reinforce voiding ability.

The various methods of bladder drainage are well known and have not changed for many decades. Such changes as have occurred are in technique of application and in the materials used, but the method of application is far more important than the method itself. A common error is the comparison of the good results obtained by one method with the bad results obtained by another. The relative merits have frequently been discussed and will not be repeated apart from comments on personal experiences of some of the main types.

Non-drainage Programme.-Overflow is a poor method because of the distention which it permits whether or not drainage is assisted by manual compression. In high cord lesions rupture of the bladder has occurred and in low cord and cauda equina lesions vesico ureteric reflux and hydronephrosis are early in evidence. In the early stages it can only be regarded as a form of retention with expressible overflow of varying amount, and few patients will tolerate Crede pressure for some weeks after a lumbar spinal injury. It is, however, a well-established method in the later 
stages of recovery when some balance of voiding ability has been achieved after a period of drainage by a catheter.

Drainage by Supra Pubic Catheter.-The procedure of suprapubic cystostomy upsets the anatomical and physiological status of the bladder. When properly performed in the dome of the bladder it does not necessarily prevent the restoration of bladder function, but the optimum time for its removal cannot be easily observed and trial periods without the drainage tube cannot be safely carried out without leakage.

Periods of catheter drainage are often necessary while the sinus heals and reinforcement mechanisms are delayed until the abdominal wall is intact. The incidence of calculi tends to be high and the ease with which the bladder is drained too often leads to a false sense' of efficiency. Many suprapubic cystostomies have been maintained until chronic infection, fibrosis and bladder contracture were irreversibly established. It is indicated where urethral pathology prevents the introduction of an indwelling catheter and it probably still has its place in battle casualties. It should, however, only be regarded as a temporary measure to be replaced as soon as expedient and certainly before the essential observations and trials of balance of bladder function are due to commence.

Drainage by Indwelling Urethral Catheter.-The indwelling urethral catheter was originally condemned as a potent cause of infection, particularly urethral and genital sepsis. It is now widely established as part of an accepted programme, especially in the spinal centres. With it, bladder capacity can be strictly controlled and distention easily avoided. Regular bladder exercise can be given and the interior of the cavity kept clear of debris by lavage. Strict asepsis can keep lower urinary tract and genital sepsis at a minimum, and at all times frequent and easy observations can be made on the efficiency of the bladder musculature.

The Catheter.-The latex rubber catheter is far better than the old rubber type formerly used. The ideal catheter is the self-retaining Foley type, usually 16 or 18 French gauge. This is well tolerated and allows any urethral discharge to escape down the urethra alongside the catheter. Meatotomy or dorsal slit are considered when necessary to avoid the train of infection which might be set up by a tight prepuce or meatus. The catheter should be changed each week, irrespective of whether it is clean or dirty. To leave it in longer increases the liability of encrustation on the catheter tip and on the balloon in particular. On deflation of the latter, phosphatic debris may be left in the bladder base, thus providing a focus of infection and stone formation.
The aseptic handling of the catheter cannot be over-emphasized. Catheterization is treated as a surgical operation and inserted with the use of sterile gloves, gowns, towels and forceps. The meticulous attention to details of asepsis is a most vital part of this method.

The catheter is attached to a closed drainage system and the urine allowed to flow freely into a bottle beneath the bed, except at times when drainage is being controlled by the use of a clip on the tubing during the later stages of bladder retraining.

Irrigation.-During its period of inactivity the bladder does not lie completely empty. At this stage it is atonic and rather like an old deflated rubber balloon. For this reason a method of lavage is employed which is certain to empty the bladder cavity at each operation. This can be achieved by suction with a Canny Ryall syringe. Twice daily the bladder can be cleared of debris, distended with half a pint of solution, and then sucked clear of all fluid by the syringe. An ideal solution for this use is Suby's solution M, which has a $\mathrm{pH}$ of $5 \cdot 5$.

Manual Control of Catheter Drainage.-This is obtained by a screw clip applied to the drainage tube and operated by the patient. It is employed as soon as the general state permits and when the stage of inactivity has passed. Normal or pero verted sensations of fullness can be recognize and the patient can practise voiding by abdominat straining or manual compression. This is pars ticularly useful in low cord or cauda equinà injuries.

Trial Periods witheut the Catheter.-Each week, when it has been removed for the routine changing, the catheter is left out for a trial period of several hours, during which observations are made on the ability to void urine. As soon as this occurs the trials are continued at weekly intervals but over longer periods, at the end of each the residual urine is withdrawn and the amount measured. The volume of residual urine depends on the efficiency of the voiding power of the bladder, and the trials are continued until the residual urine is less than the amount voided at any one time without the patient suffering any constitutional upset. At this stage the trials without a catheter can be prolonged over several days with a careful check on fluid balance and general and febrile state of the patient.

A state of balanced function may be obtained in a few weeks in incomplete neurological lesions, but anything from ro to 30 weeks may be needed in the case of complete cord or cauda equina lesions.

Therapeutics.-Antibiotics are best reserved for acute infections with constitutional disturbance. 
Mild upsets often respond to urine acidifying treatment and all cases benefit from a high fluid intake of the order of five to six pints daily. Intra-muscular injection or oral therapy with carbochol are given to most patients at the times of the trial periods without a catheter. The size of the dose and its frequency can be regulated according to the responses obtained and discontinued altogether as soon as the natural compensatory mechanisms have become established.

Instruction of the Patient.-Patients must be given some instruction on their respective bladder functions, stressing the importance of recognizing dulled or perverted sensations of impending bladder activity, of reinforcing voiding by the use of straining or manual compression and of the value of different body positions. It may be noted that in high cord lesions relaxation and steady respiration may allow more efficient voiding than by straining. These features can only be recognized by systematic observation and cooperation of all concerned and the particular method adopted must be repeated at all trial periods without a catheter and subsequently. Advice on the control of voiding by regulation and timing of the fluid intake are also invaluable. Indeed, attention to this aspect may provide the difference between uncontrolled micturition and all grades of controlled compensatory voiding.

\section{Special Investigations}

Cystometry.-This provides information on return of reflux detrusor action. It does not, however, give information concerning sphincter action nor balance of detrusor contraction and sphincter relaxation which is more important. It is therefore only used when the clinical evidence is equivocal.

Cystography.-This gives valuable information on the state of the bladder neck and presence of vesico ureteric reflux. It is not employed routinely but only in cases where balance of function is long delayed or where repeated clinical upset suggests further urinary tract pathology.

Excretion Urography.-This is employed routinely on all cases.

Cystoscopy.-Both direct vision and lens-instru ment cystoscopy are performed also on all cases. Special attention is paid to the state of the region of the external sphincter and to the bladder neck. Spasm at the level of the external sphincter may occur as part of a mass somatic reflex. A small proportion of cases benefit by pudendal neurectomy in these circumstances. Bladder neck contracture and thickening of the rim of the internal sphincter are much less common than thought hitherto. Cases presenting with these features, particularly those as a result of low cord and cauda equina injuries, benefit by transurethral resection of the bladder neck. The object again is to improve the bladder balance by lessening resistance at the internal urethral orifice. Cystoscopy is best performed at the end of a period of catheter drainage, at which time small solid debris can be removed. The operation is repeated as required in accordance with alterations in the state of bladder function.

\section{General Observations}

The improved results of bladder management are reflected in the equally improved successes of the treatment of the patient as a whole. This statement is just as true when put the other way round. The great reduction in the incidence of complications, the early mobilization of the patient and the control of infection are largely responsible for the attainment of compensatory bladder functions which are physiologically satisfactory. In the spinal centres the management of many cases by the same staff has facilitated comparative observations and standard procedures, while the instruction of the patient has undoubtedly assisted in the achievement of types of micturition which are socially safe. This state of affairs can be attained in a very high proportion of all incomplete cord lesions, but there is still much to be done before the same can be said about the results in complete cord lesions. Nevertheless, the degree of potential incontinence is continually diminishing and provides the indication that co-ordination, co-operation and meticulous attention to detail are more than worthwhile when dealing with those unfortunate enough to sustain a paraplegic disability.

This article is composed of material and abstracts taken from a paper read at the annual meeting of the Section of Urology of the Royal Society of Medicine in June I955, and published in the British Yournal of Urology of March 1956 and also an article on the Early Treatment of the Bladder in Traumatic Paraplegia published in the Fournal of Bone and Foint Surgery in August 1954.

The kind permission of the respective Editors is gratefully acknowledged. 